\title{
A Frequency Analysis Method Based On Space Beam Structure with Interval Parameters
}

\author{
Mo Yan-yu ${ }^{1, a}$, Guo Shu-xiang ${ }^{2}$, Tang Cheng ${ }^{2}$ \\ ${ }^{1}$ Aeronautics and Astronautics Engineering College, Air Force Engineering University, \\ Xi'an 710038, China \\ ${ }^{2}$ Science College, Air Force Engineering University, Xi'an 710051, China \\ ae-mail: 14031875@qq.com
}

Keywords: Uncertain structure · Frequency analysis · Frequency sensitivity · Interval parameter

\begin{abstract}
The paper aims at the frequency analysis problem of the uncertain structure, takes the space beam structure with interval parameters as the research object. An expression of the relationship between the structural natural frequencies and the interval parameters is presented. With the partial derivative of the frequency in this expression, the frequency sensitivity of interval parameters is obtained. On the basis, a frequency analysis method is presented which built on the discriminant of the sensitivity monotonicity. Use this method, a frequency analysis is carried on a simplified GARTEUR plane model. The method is then verified by using an optimization algorithm. The frequency analysis method presented in this paper has a certain theoretical and practical significance in the dynamic analysis of uncertain structure.
\end{abstract}

\section{Introduction}

Uncertainty is pervasive in the engineering practice. There must be a large amount of data for the traditional random and fuzzy method to solve the problems of uncertainty[1], otherwise the distribution functions or membership functions of the uncertain parameters will be a lack of credibility. The assumption of the distribution functions or membership functions is reasonable or not will greatly influence the outcome of the structural analysis[2]. In the engineering practice, it is always at the expense of economy to get enough data, sometimes it is even difficult to get any data, so the traditional random and fuzzy methods have certain limitations. Although it is sometimes difficult to get the accurate value of the parameter, it is much easier to obtain the interval range of the parameter. So that we can set up an interval model by limiting the uncertain parameters in specific intervals. It's an effective way to solve the uncertain problems which has a little data.

Frequency analysis is an important part of the dynamic analysis. For uncertain structure with interval parameters, the main purpose of the frequency analysis is to get the range of the natural frequency. The methods to obtain the frequency range mainly include the global optimization algorithm[3], the interval factor method based on the generalized Rayleigh quotient[4] and a method of interval Taylor expansion[5] and so on. In this paper, an expression for the relationship between the frequencies and parameters is presented. By means of the deduction of the frequency sensitivity monotonicity, the certain values of the interval parameters by which we can get the frequency extremes are obtained. The algorithm is then verified to be effective by means of the APSO intelligent algorithm. 


\section{Frequency Sensitivity Analysis of the Space Beam Structure}

Take the free vibration of the uncertain structure with n degrees of freedom into consideration. The square of the natural frequency can be obtained by solving the following generalized Rayleigh quotient $^{[4]}$

$$
\lambda_{k}=\frac{\boldsymbol{\varphi}_{k}^{T} \boldsymbol{K}(\boldsymbol{\delta}) \boldsymbol{\varphi}_{k}}{\boldsymbol{\varphi}_{k}^{T} \boldsymbol{M}(\boldsymbol{\delta}) \boldsymbol{\varphi}_{k}}
$$

where $\boldsymbol{K}(\boldsymbol{\delta})=\left[k_{i j}(\boldsymbol{\delta})\right](i, j=1,2, \ldots, n)$ denotes the general stiffness matrix, it's a $n$ orders symmetric positive semi-definite matrix; $\boldsymbol{M}(\boldsymbol{\delta})=\left[m_{i j}(\boldsymbol{\delta})\right](i, j=1,2, \ldots, n)$ denotes the general mass matrix, it's a $n$ orders symmetric positive definite matrix; $\lambda_{k}$ denotes the $k$ th order eigenvalue, it is the square of the $k$ th order natural frequency; $\boldsymbol{\varphi}_{k}$ denotes the $k$ th order eigenvector; $\delta=\left\{\delta_{i}\right\}(i=1,2, \ldots, m)$ denotes the $m$ interval variables which are unrelated to each other. Obviously, the general stiffness matrix and the general mass matrix are interval matrices. Therefore, the natural frequencies will be certain intervals.

\section{The Natural Frequency of the Space Beam Structure}

Taking the Euler-Bernoulli space beam structure with uncertain parameters into consideration. Assuming that the size parameters can be accurately obtained, and the material parameters are seen as interval variables. The structure can be simplified into $n$ elements of space beam by using finite element method(FEM). The stiffness matrix and mass matrix of each element are split into several parts. Then the general stiffness matrix and general mass matrix can be expressed as follows:

$$
\begin{aligned}
& \boldsymbol{K}=\sum_{i=1}^{n} \boldsymbol{K}^{e i}=\sum_{i=1}^{n} E_{i} A_{i} \boldsymbol{K}_{1}^{e i}+\sum_{i=1}^{n} E_{i} I_{z i} \boldsymbol{K}_{2}^{e i}+\sum_{i=1}^{n} E_{i} I_{y i} \boldsymbol{K}_{3}^{e i}+\sum_{i=1}^{n} G_{i} J_{i} \boldsymbol{K}_{4}^{e i} . \\
& \boldsymbol{M}=\sum_{i=1}^{n} \boldsymbol{M}^{e i}=\sum_{i=1}^{n} \rho_{i} A_{i} \boldsymbol{M}_{1}^{e i}+\sum_{i=1}^{n} \rho_{i} J_{i} \boldsymbol{M}_{2}^{e i} .
\end{aligned}
$$

where $A_{i}, I_{z i}, I_{y i}, J_{i}, L_{i}$ denote the certain size parameters of the $i$ th element, $\boldsymbol{K}_{1}^{e i}, \boldsymbol{K}_{2}^{e i}, \boldsymbol{K}_{3}^{e i}, \boldsymbol{K}_{4}^{e i}$, $\boldsymbol{M}_{1}^{e i}, \quad \boldsymbol{M}_{2}^{e i}$ denote certain matrices of the $i$ th element, and $E_{i}, \mu_{i}, \rho_{i}$ denote the interval material parameters of the $i$ th element. For isotropic material, $G=E /(2(1+\mu))$ holds. According to the Eq.1, the relation between the square of the structure natural frequencies and the interval variables can be deduced as follows:

$$
\begin{gathered}
\lambda_{k}=\frac{\boldsymbol{\varphi}_{k}^{T}\left(\sum_{i=1}^{n} E_{i} A_{i} \boldsymbol{K}_{1}^{e i}+\sum_{i=1}^{n} E_{i} I_{z i} \boldsymbol{K}_{2}^{e i}+\sum_{i=1}^{n} E_{i} I_{y i} \boldsymbol{K}_{3}^{e i}+\sum_{i=1}^{n} G_{i} J_{i} \boldsymbol{K}_{4}^{e i}\right) \boldsymbol{\varphi}_{k}}{\boldsymbol{\varphi}_{k}^{T}\left(\sum_{i=1}^{n} \rho_{i} A_{i} \boldsymbol{M}_{1}^{e i}+\sum_{i=1}^{n} \rho_{i} J_{i} \boldsymbol{M}_{2}^{e i}\right) \boldsymbol{\varphi}_{k}}=\frac{\boldsymbol{\varphi}_{k}^{T}\left(\sum_{i=1}^{n} E_{i} A_{i} \boldsymbol{K}_{1}^{e i}\right) \boldsymbol{\varphi}_{k}}{\boldsymbol{\varphi}_{k}^{T}\left(\sum_{i=1}^{n} \boldsymbol{M}^{\boldsymbol{e}}\right) \boldsymbol{\varphi}_{k}}+\frac{\boldsymbol{\varphi}_{k}^{T}\left(\sum_{i=1}^{n} E_{i} I_{z i} \boldsymbol{K}_{2}^{e i}\right) \boldsymbol{\varphi}_{k}}{\boldsymbol{\varphi}_{k}^{T}\left(\sum_{i=1}^{n} \boldsymbol{M}^{e i}\right) \boldsymbol{\varphi}_{k}} \\
+\frac{\boldsymbol{\varphi}_{k}^{T}\left(\sum_{i=1}^{n} E_{i} I_{y i} \boldsymbol{K}_{3}^{e i}\right) \boldsymbol{\varphi}_{k}}{\boldsymbol{\varphi}_{k}^{T}\left(\sum_{i=1}^{n} \boldsymbol{M}^{e i}\right) \boldsymbol{\varphi}_{k}}+\frac{\boldsymbol{\varphi}_{k}^{T}\left(\sum_{i=1}^{n} G_{i} J_{i} \boldsymbol{K}_{4}^{e i}\right) \boldsymbol{\varphi}_{k}}{\boldsymbol{\varphi}_{k}^{T}\left(\sum_{i=1}^{n} \boldsymbol{M}^{e i}\right) \boldsymbol{\varphi}_{k}}=\sum_{i=1}^{n} E_{i} \omega_{1 k}^{i}+\sum_{i=1}^{n} E_{i} \omega_{2 k}^{i}+\sum_{i=1}^{n} E_{i} \omega_{3 k}^{i}+\sum_{i=1}^{n} \frac{E_{i}}{2\left(1+\mu_{i}\right)} \omega_{4 k}^{i}
\end{gathered}
$$

where $\quad \omega_{1 k}^{i}=\frac{\boldsymbol{\varphi}_{k}^{T}\left(A_{i} \boldsymbol{K}_{1}^{e i}\right) \boldsymbol{\varphi}_{k}}{\boldsymbol{\varphi}_{k}^{T}\left(\sum_{i=1}^{n} \boldsymbol{M}^{\boldsymbol{e}}\right) \boldsymbol{\varphi}_{k}}, \quad \omega_{2 k}^{i}=\frac{\boldsymbol{\varphi}_{k}^{T}\left(I_{z i} \boldsymbol{K}_{2}^{e i}\right) \boldsymbol{\varphi}_{k}}{\boldsymbol{\varphi}_{k}^{T}\left(\sum_{i=1}^{n} \boldsymbol{M}^{\boldsymbol{e i}}\right) \boldsymbol{\varphi}_{k}}, \quad \omega_{3 k}^{i}=\frac{\boldsymbol{\varphi}_{k}^{T}\left(I_{y i} \boldsymbol{K}_{3}^{e i}\right) \boldsymbol{\varphi}_{k}}{\boldsymbol{\varphi}_{k}^{T}\left(\sum_{i=1}^{n} \boldsymbol{M}^{\boldsymbol{e} i}\right) \boldsymbol{\varphi}_{k}}, \quad \omega_{4 k}^{i}=\frac{\boldsymbol{\varphi}_{k}^{T}\left(J_{i} \boldsymbol{K}_{4}^{e i}\right) \boldsymbol{\varphi}_{k}}{\boldsymbol{\varphi}_{k}^{T}\left(\sum_{i=1}^{n} \boldsymbol{M}^{\boldsymbol{e i}}\right) \boldsymbol{\varphi}_{k}}$ denotes the frequency components of the $i$ th element. 


\section{Frequency Sensitivity of the Interval Variables}

Solving the partial derivative of the uncertain parameters in Eq.4, we can get the frequency sensitivity of the interval variables in the $i$ th beam element as follows:

$$
\begin{aligned}
& \frac{\partial \lambda_{k}}{\partial E_{i}}=\omega_{1 k}^{i}+\omega_{2 k}^{i}+\omega_{3 k}^{i}+\frac{\omega_{4 k}^{i}}{2\left(1+\mu_{i}\right)}, \frac{\partial \lambda_{k}}{\partial \mu_{i}}=-\frac{E_{i}}{2\left(1+\mu_{i}\right)^{2}} \omega_{4 k}^{i} \\
& \frac{\partial \lambda_{k}}{\partial \rho_{i}}=-\left(\sum_{i=1}^{n} E_{i} \omega_{1 k}^{i}+\sum_{i=1}^{n} E_{i} \omega_{2 k}^{i}+\sum_{i=1}^{n} E_{i} \omega_{3 k}^{i}+\sum_{i=1}^{n} \frac{E_{i}}{2\left(1+\mu_{i}\right)} \omega_{4 k}^{i}\right) \frac{\boldsymbol{\varphi}_{k}^{T}\left(A_{i} \boldsymbol{M}_{1}^{e i}+J_{i} \boldsymbol{M}_{2}^{e i}\right) \boldsymbol{\varphi}_{k}}{\boldsymbol{\varphi}_{k}^{T}\left(\sum_{i=1}^{n} \boldsymbol{M}^{e i}\right) \boldsymbol{\varphi}_{k}}
\end{aligned}
$$

The frequency sensitivity can be obtained by substituting the parameters into the Eq.5.

\section{Sensitivity Monotonicity Method of the Frequency Analysis}

\section{Discriminant of the Sensitivity Monotonicity}

In Eq. 4 and Eq. 5, the general mass matrix is positive definite, the size parameters $A_{i}, I_{z i}, I_{y i}, J_{i}$, $L_{i}$ are certain positives and the expressions of the certain matrices $\boldsymbol{K}_{1}^{e i}, \boldsymbol{K}_{2}^{e i}, \boldsymbol{K}_{3}^{e i}, \boldsymbol{K}_{4}^{e i}, \boldsymbol{M}_{1}^{e i}, \boldsymbol{M}_{2}^{e i}$ can be calculated by the FEM, they are omitted here due to the space limitations.

According to the knowledge of linear algebra, a sufficient and necessary condition of positive semi-definite for the real symmetric matrices is that all of the principal minors are nonnegative. Verified by calculations, the nonzero elements principal minors of $\boldsymbol{K}_{1}^{e i}$ and $\boldsymbol{K}_{4}^{e i}$ are all positives, and the nonzero elements principal minors of $\boldsymbol{K}_{2}^{e i}$ and $\boldsymbol{K}_{3}^{e i}$ are all nonnegative numbers, therefore, the matrices $\boldsymbol{K}_{1}^{e i}, \quad \boldsymbol{K}_{2}^{e i}, \quad \boldsymbol{K}_{3}^{e i}, \quad \boldsymbol{K}_{4}^{e i}$ are turned out to be positive semi-definite matrices.

An another sufficient and necessary condition of positive semi-definite for the real symmetric matrices is that any one of the highest order nonsingular principal submatrices is positive definite matrix[6]. Verified by the calculations, $\boldsymbol{M}_{1}^{e i}$ and $\boldsymbol{M}_{2}^{e i}$ are turned out to be positive semi-definite matrices in line with the condition.

In conclusion, it can be worked out that $\omega_{1 k}^{i}, \omega_{2 k}^{i}, \omega_{3 k}^{i}, \omega_{4 k}^{i}$ in Eq. 5 are all nonnegative. And then it can be obtained that the frequency sensitivity $\partial \lambda_{k} / \partial E_{i}$ is always nonnegative, $\partial \lambda_{k} / \partial \rho_{i}$ is always nonpositive and $\partial \lambda_{k} / \partial \mu_{i}$ is always nonpositive.

\section{The Calculation of the Frequency Extremes}

According to the monotonicity of the frequency sensitivity, the frequencies will achieve the maximum value when the material parameters $\rho, \mu$ get the lower bounds of the intervals and meanwhile the material parameter $E$ gets the upper bound of the interval, on the contrary, the frequencies will achieve the minimum value when the material parameters $\rho$, $\mu$ get the upper bounds of the intervals and meanwhile the material parameter $E$ gets the lower bound of the interval.

Then, the square of the frequency extremums can be obtained by Eq. 1:

$$
\lambda_{k \max }=\frac{\boldsymbol{\varphi}_{k}^{T} \boldsymbol{K}\left(\bar{E}_{i}, \underline{\mu}_{i}\right) \boldsymbol{\varphi}_{k}}{\boldsymbol{\varphi}_{k}^{T} \boldsymbol{M}\left(\underline{\rho}_{i}\right) \boldsymbol{\varphi}_{k}}, \quad \lambda_{k \min }=\frac{\boldsymbol{\varphi}_{k}^{T} \boldsymbol{K}\left(\underline{E}_{i}, \bar{\mu}_{i}\right) \boldsymbol{\varphi}_{k}}{\boldsymbol{\varphi}_{k}^{T} \boldsymbol{M}\left(\bar{\rho}_{i}\right) \boldsymbol{\varphi}_{k}}
$$

where $\bar{E}_{i}, \quad \bar{\rho}_{i}, \quad \bar{\mu}_{i}$ respectively denotes the upper bounds of the parameter intervals and $\underline{E}_{i}, \underline{\varrho}_{i}$, $\underline{\mu}_{i}$ denotes the lower bounds of the parameter intervals.

The Optimization Algorithm to Calculate the Frequency Range

Considering that the eigenvalue $\lambda_{k}$ in Eq. 1 is an interval number, generally we can solve the generalized eigenvalue problem with a global optimization method. An optimization model can be established as follows: 


$$
\begin{aligned}
& \min \text { and } \max \lambda_{k}=\frac{\boldsymbol{\varphi}_{k}^{T} \boldsymbol{K} \boldsymbol{\varphi}_{k}}{\boldsymbol{\varphi}_{k}^{T} \boldsymbol{M} \boldsymbol{\varphi}_{k}} \\
& \text { s.t. }\left\{\begin{array}{l}
\boldsymbol{K}=\boldsymbol{K}(\boldsymbol{\delta}), \boldsymbol{M}=\boldsymbol{M}(\boldsymbol{\delta}), \boldsymbol{\delta}=\left\{\delta_{i}\right\}, \\
\delta_{i}^{l} \leq \delta_{i} \leq \delta_{i}^{u}, i=1,2, \cdots, m .
\end{array}\right.
\end{aligned}
$$

where $\boldsymbol{\delta}=\left\{\delta_{i}\right\} \quad(i=1,2, \ldots, m)$ denotes the $m$ interval optimization variables, $\delta_{i}^{l}$ denotes the lower bound of the interval variables and $\delta_{i}^{u}$ denotes the upper bound of the interval variables. Using this model, the upper and lower bounds of the eigenvalues can be obtained, thus, the natural frequency intervals can be solved out.

Theoretically, the optimization algorithm can solve the problem of frequency interval well. The genetic algorithm in the literature [3] obtains much better results than any other numerical algorithms do when calculating the interval eigenvalues. In this paper, an adaptive particle swarm optimization algorithm (APSO) is used for the optimization calculation. The APSO algorithm could automatically change the setting of the acceleration factor and the inertia weight as the iteration progresses, so that it has better global optimization ability in the earlier iterations and has better convergence speed in the late iterations. Therefore, the efficiency is significantly improved.

\section{Numerical Example}

GARTEUR plane model is a benchmark model[7]. It was designed by the French Aerospace Research Institute in 1995[8]. The model has the same characteristic with the real aircraft such as high flexibilities, modal density and so on. As shown in Fig. 1(a), the model has a total length of $1.5 \mathrm{~m}$, a wingspan of $2.0 \mathrm{~m}$, the material is aluminum, there is a damping layer attaching to the wing. The density, elastic modulus and poisson ratio of the wing changed due to the existence of damping

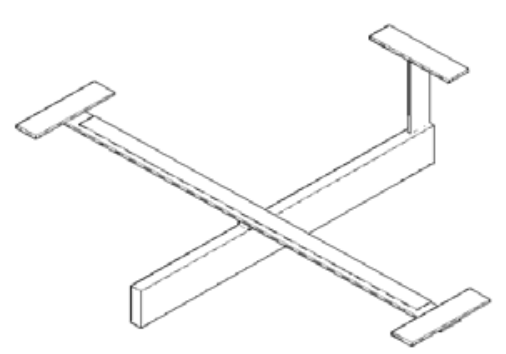

(a) GARTEUR Plane Model

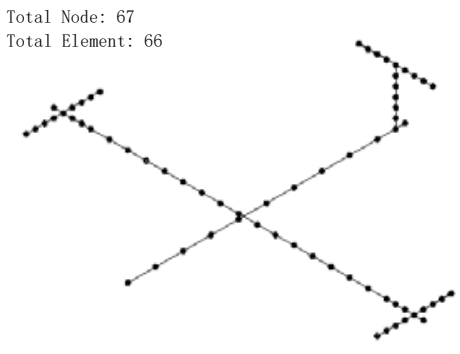

(b) Finite Element Model

Fig.1 A Simplified GARTEUR Model

layer[7, 9]. The whole model is simplified to a space beam model, and there are a total of 67 nodes and 66 beam elements, the specific model is shown in Fig. 1(b). Considering the simplification of the connection between the wing and the fuselage, set the elastic modulus of the connection element as interval variable $E_{l}$, with the range of $[100,1000] G P a$. Considering the existence of the damping layer, set the elastic modulus of the wing as interval variable $E_{2 c}$, with the range of $[67.5,68.5] G P a$, the density of the wing as interval variable $\rho_{2 c}$, with the range of $[2700,2900] \mathrm{Kg} / \mathrm{m}^{3}$, the poisson ratio of the wing as interval variable $\mu_{2 c}$, with the range of $[0.29,0.31]$.

By use of the sensitivity monotonicity algorithm and the APSO algorithm, the frequency analysis is respectively carried out on the GARTEUR plane model. The frequency intervals are respectively obtained as a result. Takes the first 6 order frequency intervals be shown in Table 1. From Table 1 we can find that the results of the two algorithms are nearly the same, which shows that the sensitivity monotonicity algorithm is valid. In addition, the sensitivity monotonicity algorithm doesn't need iteration, so it has much faster computing speed. 
Table 1 The Natural Frequency Intervals of GARTEUR Model

\begin{tabular}{ccc}
\hline Frequency & Monotonicity Algorithm & APSO Algorithm \\
\hline$\left[\lambda_{1}^{l}, \lambda_{1}^{u}\right]$ & {$[5.1252,5.2395]$} & {$[5.1252,5.2393]$} \\
{$\left[\lambda_{2}^{l}, \lambda_{2}^{u}\right]$} & {$[5.1954,5.2452]$} & {$[5.1955,5.245]$} \\
{$\left[\lambda_{3}^{l}, \lambda_{3}^{u}\right]$} & {$[24.2211,24.2211]$} & {$[24.2211,24.2211]$} \\
{$\left[\lambda_{4}^{l}, \lambda_{4}^{u}\right]$} & {$[33.4799,35.495]$} & {$[33.4799,35.4919]$} \\
{$\left[\lambda_{5}^{l}, \lambda_{5}^{u}\right]$} & {$[38.8969,39.3312]$} & {$[38.8971,39.3310]$} \\
{$\left[\lambda_{6}^{l}, \lambda_{6}^{u}\right]$} & {$[41.1152,42.6744]$} & {$[41.1160,42.6744]$} \\
\hline
\end{tabular}

\section{Conclusion}

Frequency analysis is an important part of the structure dynamic analysis. The paper aims at the frequency analysis problem of the uncertain structure, tries to calculate the natural frequencies sensitivity of the uncertain space beam structure on the theoretical basis of the finite element method, and then attempts to obtained the frequency intervals by use of the sensitivity monotonicity algorithm. The algorithm is verified by the APSO global optimization algorithm then. After theoretical deduction, practical calculation and optimization algorithm verification, we can find that the sensitivity monotonicity algorithm is effective and useful for solving the frequency analysis problems of the space beam structure while the material parameters are assumed as interval variables and the size parameters are certain values.

\section{Acknowledgements}

The paper was supported by the National Natural Science Foundation of China (Grant no. 51175510).

\section{References}

[1] Elishkaoff I. Essay on uncertainties in elastic and viscoelastic structures: from A. M. Freudenthal's criticisms to modern convex modeling[J]. Computers \& Structures, 56(6), 1995: 871-895.

[2] Ben-haim Y, Elishakoff I. Convex Models of Uncertainty in Applied Mechanics[J]. Amsterdam: Elsevier Science Publisher, 1990.

[3] Wang Denggang. Global optimization method for computing frequencies of structures with interval uncertain parameters[J]. ACTA MECHANICA SINICA, 2004, 36(3): 364-372. (In Chinese)

[4] YANG Yu-jun, CHEN Jian-jun. A fast method for computing eigenvalue of a structural matrix with interval parameters[J]. Journal of Vibration and Shock, 2009, 28(1):42-45. (In Chinese)

[5] MA Liang, CHEN Su-huan, MENG Guang-wei. Eigenvalue analysis of structures with large variations of interval parameters[J]. Journal of Jilin University(Engineering and Technology Edition), 2009, 39(1): 98-102. (In Chinese)

[6] Song Dao-jin. A criterion theorem of real symmetric positive semidefinite matrix[J]. Journal of Qufu Normal University(Natural Science), 1990, 3: 44. (In Chinese)

[7] Bi S F, Deng Z M. Stochastic model updating and validation of the GARTEUR structure based on random sampling and distance discrimination[J]. Acta Aeronautica et Astronautica Sinica, 2013, 34(12): 2757-2767. (In Chinese) 
[8] Hilde De Gersem, David Moens, Wim Desmet, Dirk Vandepitte. Interval and fuzzy dynamic analysis of finite element models with superelements[J]. Computers \& Structures, 2007, 85: 304-319.

[9] Degener M, Hermes M. Ground vibration test and finite element analysis of the GARTEUR SM-AG19 testbed. DLR Report, IB 232-96 J08,1996. 